\title{
Pascu-Type Analytic Functions by Using Mittag-Leffler Functions in Janowski Domain
}

\author{
Wali Khan Mashwan $\left(\mathbb{D},{ }^{1}\right.$ Bakhtiar Ahmad ${ }^{D},{ }^{2}$ Muhammad Ghaffar Khan ${ }^{(D)}$, \\ Saima Mustafa, ${ }^{3}$ Sama Arjika ${ }^{(D)},{ }^{4,5}$ and Bilal Khan ${ }^{6}$ \\ ${ }^{1}$ Institute of Numerical Sciences, Kohat University of Science and Technology, Kohat, Pakistan \\ ${ }^{2}$ Govt: Degree College Mardan, Mardan 23200, Pakistan \\ ${ }^{3}$ Department of Mathematics and Statistics, PMAS Arid Agriculture University, Rawalpindi, Pakistan \\ ${ }^{4}$ Department of Mathematics and Informatics, University of Agadez, Agadez, Niger \\ ${ }^{5}$ International Chair of Mathematical Physics and Applications (ICMPA-UNESCO Chair), University of Abomey-Calavi, \\ Post Box 072, Cotonou 50, Benin \\ ${ }^{6}$ School of Mathematical Sciences and Shanghai Key Laboratory of PMMP, East China Normal University, 500 Dongchuan Road, \\ Shanghai 200241, China
}

Correspondence should be addressed to Sama Arjika; rjksama2008@gmail.com

Received 8 May 2021; Revised 5 July 2021; Accepted 30 July 2021; Published 12 August 2021

Academic Editor: A. M. Bastos Pereira

Copyright (C) 2021 Wali Khan Mashwan et al. This is an open access article distributed under the Creative Commons Attribution License, which permits unrestricted use, distribution, and reproduction in any medium, provided the original work is properly cited.

Keeping in view the various important applications of Mittag-Leffer functions in the fields of applied sciences, we introduce Pascutype analytic functions utilizing the concept of Mittag-Leffler functions in the region of Janowski domain. Moreover, we investigate some useful properties of these functions such as sufficiency criteria, distortion and growth bounds, convex combination, radius of starlikeness, and some partial sum results.

\section{Introduction, Definitions, and Motivation}

Let $\mathfrak{A}$ denote the class of functions $f$ that are analytic in the open unit disc $\mathbb{D}=\{z \in \mathbb{C}:|z|<1\}$, and its Taylor series representation is as follows:

$$
f(z)=z+\sum_{k=2}^{\infty} a_{k} z^{k}, \quad(z \in \mathbb{D})
$$

Also, the most well-known subclass of $\mathfrak{A}$ is the class of univalent functions denoted by $\delta$.

Further, $\mathcal{S}^{*}$ represents the class of starlike functions in $\mathbb{D}$, that is, $f \in \mathcal{S}$ and maps $\mathbb{D}$ on an starlike domain. Mathematically, it will satisfy the following condition:

$$
\mathfrak{R}\left\{\frac{z f^{\prime}(z)}{f(z)}\right\}>0, \quad(z \in \mathbb{D}) .
$$

Now let us recall the familiar concept of subordinations; let $h_{1}$ and $h_{2}$ be two functions of class $\mathfrak{A}$, and we call that a function $h_{1}$ is subordinated to $h_{2}$ and symbolically represented as

$$
h_{1} \prec h_{2} \text { or } h_{1}(z) \prec h_{2}(z),
$$

if there exists Schwarz function $u$, with conditions $u(0)=0$ and $|u(z)|<1$ such that

$$
h_{1}(z)=h_{2}(u(z)) .
$$

Further if $h_{2} \in \mathcal{S}$, then the above definition is parallel to

$$
\begin{aligned}
& h_{1}(z) \prec h_{2}(z)(z \in \mathbb{D}) \Leftrightarrow h_{1}(0)=h_{2}(0), \\
& h_{1}(\mathbb{D}) \subset h_{2}(\mathbb{D}) .
\end{aligned}
$$

In 1973, Janowski [1] introduced the concepts of circular domain by introducing Janowski-type functions. A function $g(z)$ analytic in open unit disc with properties that $g(0)=1$ is said to be in the class of Janowski denoted by $P[A, B]$ if for $-1 \leq B<A \leq 1$, 


$$
g(z) \prec \frac{1+A z}{1+B z} .
$$

Janowski showed that $g(z)$ maps $\mathbb{D}$ onto domain $\Omega(A, B)$, where circular domain $\Omega(A, B)$ is defined by

$$
\Omega(A, B)= \begin{cases}\omega:\left|\omega-\frac{1-A B}{1-B^{2}}\right|<\frac{A-B}{1-B^{2}}, & \text { for } B \neq-1, \\ \omega: \mathfrak{R}(\omega)>\frac{1-A}{2}, & \text { for } B=-1 .\end{cases}
$$

The domain $\Omega(A, B), B \neq-1$, represents an open circular disc centered on real axis with $D_{1}=1-A / 1-B$ and $D_{2}=1+A / 1+B$ as diameter end points with $0<D_{1}<1<D_{2}$. After introducing circular domain's concept, several researchers focused on this domain and associated several subclasses to this domain and investigated some of its interesting geometric properties. For some recent works on Janowski-type domain, we refer the readers to [2-6].

The use of special functions in the field of mathematics and applied sciences is very important. One special-type function with single parameter $\alpha$ is defined as

$$
E_{\alpha}(z)=\sum_{k=0}^{\infty} \frac{z^{k}}{\Gamma(1+\alpha k)}, \quad \alpha, z \in \mathbb{C}, \text { and } \mathfrak{R}(\alpha)>0,
$$

and with two parameters $\alpha$ and $\beta$, its generalized form is

$$
E_{\alpha, \beta}(z)=\sum_{k=0}^{\infty} \frac{z^{k}}{\Gamma(\beta+\alpha k)}, \quad \alpha, \beta, z \in \mathbb{C} \text { and } \Re(\alpha)>0, \Re(\beta)>0,
$$

which are known as Mittag-Leffler functions (see [7]). This was introduced by Mittag-Leffler [8] in connection with his method of summation of some divergent series. Mittag-Leffler in his paper [8] examined and investigated certain very interesting properties of functions defined in form (9).

Mittag-Leffler functions naturally arise in solutions of fractional-order integral equations or fractional-order differential equations. Especially these type of functions can be seen in the investigations of the fractional generalization of the kinetic equation, Levy flights, random walks, superdiffusive transport, and in the study of complex systems. Mittag-Leffler functions, both ordinary and generalized functions, interpolate between a purely exponential law and power-law-like behavior of phenomena governed by ordinary kinetic equations and fractional counterparts (see [9] for more details). During the last 15 years, the interest of Mittag-Leffler functions is considerably increased among engineers and scientists due to their vast applications in several applied problems such as fluid flow, rheology, diffusive transport akin to diffusion, electric networks, probability, and probability distribution (for more applications, see $[10,11])$. By giving specific values to $\alpha$ and $\beta$, we can get the following special cases easily.
(1)

$$
E_{0}(z)=\frac{1}{1-z}, \quad|z|<1
$$

(2)

$$
E_{1}(z)=e^{z}
$$

$$
E_{2}(z)=\cosh (\sqrt{z}), \quad z \in \mathbb{C}
$$

$$
E_{2}\left(-z^{2}\right)=\cos (z), \quad z \in \mathbb{C}
$$

$$
E_{3}(z)=\frac{1}{2}\left[e^{z^{1 / 3}}+2 e^{-(1 / 2) z^{1 / 3}} \cos \left(\frac{\sqrt{3}}{2} z^{1 / 3}\right)\right], \quad z \in \mathbb{C}
$$

Now the function is defined by

$$
Q_{\alpha, \beta}(z)=z \Gamma(\beta) E_{\alpha, \beta}(z)=z+\sum_{k=2}^{\infty} \frac{\Gamma(\beta)}{\Gamma(\beta+\alpha(k-1))} z^{k} .
$$

Related to this, Elhaddad et al. [12] defined the operator $\mathscr{D}_{\mu}^{m}(\alpha, \beta) f(z): \mathfrak{A} \longrightarrow \mathfrak{A}$ as follows:

$$
\mathscr{D}_{\mu}^{m}(\alpha, \beta) f(z)=z+\sum_{k=2}^{\infty}[1+(k-1) \mu]^{m} \frac{\Gamma(\beta)}{\Gamma(\beta+\alpha(k-1))} z^{k},
$$

where $m \in \mathbb{N}_{0}=\mathbb{N} \cup\{0\}, \mu \geq 0$.

Now let us recall some recent Pascu-type works. In 2014, Vijaya et al. [13] defined Pascu-type class for harmonic functions with positive coefficient involving Salagean operator. After that, in 2015, Murugusundaramoorty et al. [14] found coefficient estimates for Pascu-type subclass of bi-univalent functions based on subordination. Recently, Vijaya [15] obtained coefficient bounds for subclass of Pascu-type bi-starlike functions in parabolic domain.

Motivated from above and the work studied in [16-21], we now define a subfamily $\mathcal{S}_{\mu}^{m, t}(\alpha, \beta, A, B)$ of $\mathfrak{\mathcal { U }}$ by using the operator $\mathscr{D}_{\mu}^{m}$ as follows.

Definition 1. With conditions $-1 \leq B<A \leq 1, f \in \mathfrak{A}$ will be in the class $\mathcal{S}_{\mu}^{m, t}(\alpha, \beta, A, B)$, if it satisfies

$$
\frac{(1-t) \mathscr{D}_{\mu}^{m+1}(\alpha, \beta) f(z)+t \mathscr{D}_{\mu}^{m+2}(\alpha, \beta) f(z)}{(1-t) \mathscr{D}_{\mu}^{m}(\alpha, \beta) f(z)+t \mathscr{D}_{\mu}^{m+1}(\alpha, \beta) f(z)} \prec \frac{1+A z}{1+B z},
$$

where the notation “〈” stands for the familiar concept of subordinations. 
It can easily be easily verified that a function $f \in \mathfrak{A}$ will be in the class $\mathcal{S}_{\mu}^{m, t}(\alpha, \beta, A, B)$, if and only if

$$
\left|\frac{\left((1-t) \mathscr{D}_{\mu}^{m+1}(\alpha, \beta) f(z)+t \mathscr{D}_{\mu}^{m+2}(\alpha, \beta) f(z) /(1-t) \mathscr{D}_{\mu}^{m}(\alpha, \beta) f(z)+t \mathscr{D}_{\mu}^{m+1}(\alpha, \beta) f(z)\right)-1}{B\left((1-t) \mathscr{D}_{\mu}^{m+1}(\alpha, \beta) f(z)+t \mathscr{D}_{\mu}^{m+2}(\alpha, \beta) f(z) /(1-t) \mathscr{D}_{\mu}^{m}(\alpha, \beta) f(z)+t \mathscr{D}_{\mu}^{m+1}(\alpha, \beta) f(z)\right)-A}\right|<1 .
$$

\section{The Main Results and Their Consequences}

$$
\left|\frac{K(z)-H(z)}{B K(z)-A H(z)}\right|<1
$$

In this section, we start with sufficiency criteria for this newly defined class.

Theorem 1. Let $f \in \mathfrak{A}$ of form (1) be in the class $\mathcal{S}_{\mu}^{m, t}(\alpha, \beta, A, B)$ if and only if the inequality

$$
\sum_{k=2}^{\infty} \Lambda_{k} \sigma_{k}\left|a_{k}\right| \leq|B-A|
$$

is satisfied, where $\sigma_{k}=|\Gamma(\beta) / \Gamma(\alpha(k-1)+\beta)|$ and $\Lambda_{k}=$ $(1+(k-1) \eta)^{k}(1+\lambda(k-1) \eta)[(1+B)(k-1) \eta+B-A]$.

Proof. To show that $f \in \mathcal{S}_{\mu}^{m, t}(\alpha, \beta, A, B)$, it will be enough if we show that inequality (18) holds true, i.e., where

$$
\begin{aligned}
K(z) & =(1-t) \mathscr{D}_{\mu}^{m+1}(\alpha, \beta) f(z)+t \mathscr{D}_{\mu}^{m+2}(\alpha, \beta) f(z) \\
& =z+\sum_{k=2}^{\infty}(1+(k-1) \eta)^{k+1}(1+\lambda(k-1) \eta) \sigma_{k} a_{k} z^{k}, \\
H(z) & =(1-t) \mathscr{D}_{\mu}^{m}(\alpha, \beta) f(z)+t \mathscr{D}_{\mu}^{m+1}(\alpha, \beta) f(z) \\
& =z+\sum_{k=2}^{\infty}(1+(k-1) \eta)^{k}(1+\lambda(k-1) \eta) \sigma_{k} a_{k} z^{k} .
\end{aligned}
$$

Now consider

$$
\begin{aligned}
\left|\frac{K(z)-H(z)}{B K(z)-A H(z)}\right| & =\left|\frac{\sum_{k=2}^{\infty}(1+(k-1) \eta)^{k}(1+\lambda(k-1) \eta)(k-1) \eta \sigma_{k} a_{k} z^{k}}{(B-A) z+\sum_{k=2}^{\infty}(1+(k-1) \eta)^{k}(1+\lambda(k-1) \eta)(B(k-1) \eta+B-A) \sigma_{k} a_{k} z^{k}}\right| \\
& =\frac{\sum_{k=2}^{\infty}(1+(k-1) \eta)^{k}(1+\lambda(k-1) \eta)(k-1) \eta \sigma_{k}\left|a_{k}\right|}{(B-A)-\sum_{k=2}^{\infty}(1+(k-1) \eta)^{k}(1+\lambda(k-1) \eta)(B(k-1) \eta+B-A) \sigma_{k}\left|a_{k}\right|}<1 .
\end{aligned}
$$

Here we have used inequality (19), and this completes the direct part of the proof.
For converse part, let $f \in \mathcal{S}_{\mu}^{m, t}(\alpha, \beta, A, B)$ be given by (1); then, from (18), we have for $z \in \mathbb{D}$,

$$
\begin{aligned}
& \left|\frac{\left((1-t) z \mathscr{D}_{\mu}^{m+1}(\alpha, \beta) f(z)+t \mathscr{D}_{\mu}^{m+2}(\alpha, \beta) f(z) /(1-t) z \mathscr{D}_{\mu}^{m}(\alpha, \beta) f(z)+t \mathscr{D}_{\mu}^{m+1}(\alpha, \beta) f(z)\right)-1}{B\left((1-t) z \mathscr{D}_{\mu}^{m+1}(\alpha, \beta) f(z)+t \mathscr{D}_{\mu}^{m+2}(\alpha, \beta) f(z) /(1-t) z \mathscr{D}_{\mu}^{m}(\alpha, \beta) f(z)+t \mathscr{D}_{\mu}^{m+1}(\alpha, \beta) f(z)\right)-A}\right| \\
& \quad=\left|\frac{\sum_{k=2}^{\infty}(1+(k-1) \eta)^{k}(1+\lambda(k-1) \eta)(k-1) \eta \sigma_{k} a_{k} z^{k}}{(B-A) z+\sum_{k=2}^{\infty}(1+(k-1) \eta)^{k}(1+\lambda(k-1) \eta)(B(k-1) \eta+B-A) \sigma_{k} a_{k} z^{k}}\right| .
\end{aligned}
$$

Since $\mathfrak{R e}(z) \leq|z|$, we have

$$
\mathfrak{R e}\left\{\frac{\sum_{k=2}^{\infty}(1+(k-1) \eta)^{k}(1+\lambda(k-1) \eta)(k-1) \eta \sigma_{k} a_{k} z^{k}}{(B-A)+\sum_{k=2}^{\infty}(1+(k-1) \eta)^{k}(1+\lambda(k-1) \eta)(B(k-1) \eta+B-A) \sigma_{k} a_{k} z^{k}}\right\}<1 .
$$


Now we choose values of $z$ on the real axis so that $(1-t)$ $z \mathscr{D}_{\mu}^{m+1}(\alpha, \beta) f(z)+t \mathscr{D}_{\mu}^{m+2} \quad(\alpha, \beta) f(z) /(1-t) z \mathscr{D}_{\mu}^{m}(\alpha, \beta) f$ $(z)+t \mathscr{D}_{\mu}^{m+1}(\alpha, \beta) f(z)$ is real. Upon clearing the denominator in (2) and letting $z \longrightarrow 1^{-}$through real values, we obtain (19).

Example 1. For the function

$$
f(z)=z+\sum_{k=2}^{\infty} \frac{|B-A|}{\Lambda_{k} \sigma_{k}} x_{k} z^{k},
$$

where $\sigma_{k}=|\Gamma(\beta) / \Gamma(\alpha(k-1)+\beta)|$ and $\Lambda_{k}=(1+(k-1) \eta)$ ${ }^{k}(1+\lambda(k-1) \eta)[(1+B)(k-1) \eta+B-A]$, we have

Hence, $f \in \mathcal{S}_{\mu}^{m, t}(\alpha, \beta, A, B)$, and the result is sharp for this function.

$$
\begin{aligned}
\sum_{k=2}^{\infty} \Lambda_{k} \sigma_{k}\left|a_{k}\right| & =\operatorname{sum}_{n=2}^{\infty} \Lambda_{k} \sigma_{k} \frac{|B-A|}{\Lambda_{k} \sigma_{k}}\left|x_{k}\right| \\
& =|B-A| \sum_{n=2}^{\infty}\left|x_{n}\right|=|B-A| .
\end{aligned}
$$

Corollary 1. Let $f \in \mathfrak{A}$ of form (1) be in the class $\mathcal{S}_{\mu}^{m, t}(\alpha, \beta, A, B)$. Then,

$$
\left|a_{k}\right| \leq \frac{|B-A|}{\Lambda_{k} \sigma_{k}},
$$

where $\Lambda_{k}$ and $\sigma_{k}$ are given above for $k \geq 2$.

In the following, we discuss the growth and distortion theorems for our new class of functions.

Theorem 2. If $f$ is in the class $\mathcal{S}_{\mu}^{m, t}(\alpha, \beta, A, B)$ and has form (1). Then, for $|z|=r$, we have

$$
\begin{gathered}
r-\frac{|B-A|}{\Lambda_{2} \sigma_{2}} r^{2} \leq|f(z)| \leq r+\frac{|B-A|}{\Lambda_{2} \sigma_{2}} r^{2}, \\
1-\frac{2|B-A|}{\Lambda_{2} \sigma_{2}} r \leq\left|f^{\prime}(z)\right| \leq 1+\frac{2|B-A|}{\Lambda_{2} \sigma_{2}} r .
\end{gathered}
$$

These inequalities are sharp for the function defined as

$$
f(z)=z-\frac{|B-A|}{\Lambda_{2} \sigma_{2}} z^{2} .
$$

Proof. First, we want to prove inequality (28). For this, consider

$$
|f(z)|=\left|z+\sum_{k=2}^{\infty} a_{k} z^{k}\right| \leq|z|+\sum_{k=2}^{\infty}\left|a_{k}\right||z|^{k},
$$

since for $|z|=r<1$ we have $r^{n} \leq r^{2}$ for $n \geq 2$ and

$$
|f(z)| \leq r+r^{2} \sum_{k=2}^{\infty}\left|a_{k}\right|
$$

Similarly,

$$
|f(z)| \geq r-r^{2} \sum_{k=2}^{\infty}\left|a_{k}\right|
$$

Now (19) implies that

$$
\sum_{k=2}^{\infty} \Lambda_{n} \sigma_{k}\left|a_{k}\right| \leq|B-A|
$$

But

$$
\sigma_{2} \Lambda_{2} \sum_{k=2}^{\infty}\left|a_{k}\right| \leq \sum_{k=2}^{\infty} \Lambda_{k} \sigma_{k}\left|a_{k}\right|
$$

Hence,

$$
\sum_{k=2}^{\infty} \sigma_{2} \Lambda_{2}\left|a_{k}\right| \leq|B-A|
$$

which gives

$$
\sum_{k=2}^{\infty}\left|a_{k}\right| \leq \frac{|B-A|}{\Lambda_{2} \sigma_{2}} .
$$

Now by putting this value in (32) and (33), we get the required result. The proof of (29) is similar to (28), so it is omitted.

Theorem 3. The class $\delta_{\mu}^{m, t}(\alpha, \beta, A, B)$ is closed under convex combination.

Proof. To show the class $\mathcal{S}_{\mu}^{m, t}(\alpha, \beta, A, B)$ is closed under convex combination, it is enough to show that $\mu f_{1}(z)+(1-\mu) f_{2}(z) \in \mathcal{S}_{\mu}^{m, t}(\alpha, \beta, A, B)$, where $f_{1}$ and $f_{2} \in \mathcal{S}_{\mu}^{m, t}(\alpha, \beta, A, B)$ and $\mu \in[0,1]$. Since

$$
\mu f_{1}(z)+(1-\mu) f_{2}(z)=z+\sum_{k=2}^{\infty}\left(\mu a_{k, 1}+(1-\mu) a_{k, 2}\right) z^{k},
$$

by Theorem 1, we have

$$
\begin{aligned}
\sum_{k=2}^{\infty} \Lambda_{k} \sigma_{k}\left|\mu a_{k, 1}+(1-\mu) a_{k, 2}\right| & \leq \mu \sum_{k=2}^{\infty} \Lambda_{k} \sigma_{k}\left|a_{k, 1}\right|+(1-\mu) \sum_{k=2}^{\infty} \Lambda_{k} \sigma_{k}\left|a_{k, 2}\right| \\
& \leq \mu|B-A|+(1-\mu)|B-A|=|B-A| .
\end{aligned}
$$

Hence, in light of Theorem 1, it is the required result.

Theorem 4. Let

$$
f_{k}(z)=z+\frac{1}{\Lambda_{k} \sigma_{k}} z^{k}
$$


with $f_{1}(z)=z$; then, $f \in \mathcal{S}_{\mu}^{m, t}(\alpha, \beta, A, B)$ if and only if

$$
f(z)=\sum_{k=1}^{\infty} \gamma_{k} f_{k}(z), \quad \text { for } \gamma_{k} \geq 0
$$

with property that $\sum_{k=1}^{\infty} \gamma_{k}=1$.

Proof. Let (41) hold. Then,

$$
\sum_{k=2}^{\infty} \Lambda_{k} \sigma_{k}\left|a_{k}\right|=\sum_{k=2}^{\infty} \Lambda_{k} \sigma_{k}\left\{\frac{1}{\Lambda_{k} \sigma_{k}}\right\} \gamma_{k} \leq|B-A|\left(1-\gamma_{1}\right) \leq|B-A| .
$$

Hence, by Theorem 1, $f(z) \in \mathcal{S}_{\mu}^{m, t}(\alpha, \beta, A, B)$. Conversely, suppose $f(z) \in \mathcal{S}_{\mu}^{m, t}(\alpha, \beta, A, B)$; then, we have

$$
\gamma_{k}=\Lambda_{k} \sigma_{k}\left|a_{k}\right|, \quad \text { for } k \geq 2,
$$

and $\gamma_{1}=1-\sum_{k=2}^{\infty} \gamma_{k}$. Then, function is of the form given by (40), and thus this completes the proof.

Theorem 5. Let $f(z) \in \mathcal{S}_{\mu}^{m, t}(\alpha, \beta, A, B)$; then, $f$ is starlike function of order $\delta$ in $|z|<r^{*}$, where

$$
r^{*}=\left\{\frac{(1-\delta) \Lambda_{k} \sigma_{k}}{(n-\delta)|B-A|}\right\}^{1 /(n-1)}
$$

Proof. We know that $f$ is starlike function of order $\delta$ if and only if

$$
\left|\frac{z f^{\prime}(z)-f(z)}{z f^{\prime}(z)-(1-2 \delta) f(z)}\right|<1 .
$$

Using (8) and upon simplification yields

$$
\sum_{k=2}^{\infty}\left(\frac{n-\delta}{1-\delta}\right)\left|a_{k}\right||z|^{n-1} \leq 1
$$

In light of Theorem 1 , we can easily obtain

$$
\sum_{k=2}^{\infty} \frac{\Lambda_{k} \sigma_{k}}{|B-A|}\left|a_{k}\right| \leq 1
$$

which is possible if

$$
\sum_{k=2}^{\infty}\left(\frac{n-\delta}{1-\delta}\right)\left|a_{k}\right||z|^{n-1}<\sum_{k=2}^{\infty} \frac{\Lambda_{k} \sigma_{k}}{|B-A|}\left|a_{k}\right|
$$

which implies that

$$
|z|<\left\{\frac{(1-\delta) \Lambda_{k} \sigma_{k}}{(n-\delta)|B-A|}\right\}^{1 /(n-1)} .
$$

Hence, we obtain the desired result.

Theorem 6. If $f$ is in the class $\delta_{\mu}^{m, t}(\alpha, \beta, A, B)$ and has form (1). Then,

$$
\begin{aligned}
& \mathfrak{R}\left\{\frac{f(z)}{f_{m}(z)}\right\} \geq 1-\frac{1}{c_{m+1}}, \\
& \mathfrak{R}\left\{\frac{f_{m}(z)}{f(z)}\right\} \geq \frac{c_{m+1}}{1+c_{m+1}},
\end{aligned}
$$

where

$$
\begin{aligned}
c_{k}= & \frac{\Lambda_{k} \sigma_{k}}{|B-A|}, \\
& \quad c_{k} \geq\left\{.1 \text { for } k=2,3, \ldots, m c_{k+1} \text { for } k=m+1, \ldots\right\} .
\end{aligned}
$$

Proof. First we prove (50); for this, let us write

$$
c_{k+1}\left\{\frac{f(z)}{f_{m}(z)}-\left(1-\frac{1}{c_{m+1}}\right)\right\}=1+\frac{c_{k+1} \sum_{k=m+1}^{\infty} a_{k} z^{k-1}}{1+\sum_{k=2}^{m} a_{k} z^{k-1}}=\frac{1+w(z)}{1-w(z)},
$$

where

$$
\begin{gathered}
w(z)=\frac{c_{k+1} \sum_{k=m+1}^{\infty} a_{k} z^{k-1}}{2+2 \sum_{k=2}^{m} a_{k} z^{k-1}+c_{k+1} \sum_{k=m+1}^{\infty} a_{k} z^{k-1}}, \\
|w(z)| \leq \frac{c_{k+1} \sum_{k=m+1}^{\infty}\left|a_{k}\right|}{2-2 \sum_{k=2}^{m}\left|a_{k}\right|-c_{k+1} \sum_{k=m+1}^{\infty}\left|a_{k}\right|} \leq 1,
\end{gathered}
$$

if and only if

$$
\sum_{k=2}^{m}\left|a_{k}\right|+c_{k+1} \sum_{k=m+1}^{\infty}\left|a_{k}\right| \leq 1 \text {. }
$$

In order to prove the inequality in (50), it is enough to show that the left-hand side of (55) is bounded above by the following sum:

$$
\sum_{k=2}^{\infty} c_{k}\left|a_{k}\right|
$$

and is equivalent to

$$
\sum_{k=2}^{m}\left(1-c_{k}\right)\left|a_{k}\right|+c_{k+1} \sum_{k=m+1}^{\infty}\left(c_{m+1}-c_{k}\right)\left|a_{k}\right| \geq 0,
$$

which is true for inequality (50).

Next to derive inequality (51), let us write

$$
\begin{aligned}
\left(1+c_{k+1}\right)\left\{\frac{f_{m}(z)}{f(z)}-\left(1+\frac{1}{c_{m+1}}\right)\right\} & =1-\frac{\left(1+c_{k+1}\right) \sum_{k=m+1}^{\infty} a_{k} z^{k-1}}{1+\sum_{k=2}^{m} a_{k} z^{k-1}} \\
& =\frac{1+w(z)}{1-w(z)},
\end{aligned}
$$

where 


$$
\begin{gathered}
w(z)=\frac{-\left(1+c_{k+1}\right) \sum_{k=m+1}^{\infty} a_{k} z^{k-1}}{2+2 \sum_{k=2}^{m} a_{k} z^{k-1}-\left(1+c_{k+1}\right) \sum_{k=m+1}^{\infty} a_{k} z^{k-1}}, \\
|w(z)| \leq \frac{\left(1+c_{k+1}\right) \sum_{k=m+1}^{\infty}\left|a_{k}\right|}{2-2 \sum_{k=2}^{m}\left|a_{k}\right|-\left(1+c_{k+1}\right) \sum_{k=m+1}^{\infty}\left|a_{k}\right|} \leq 1
\end{gathered}
$$

is equivalent to the following inequality:

$$
\sum_{k=2}^{m}\left|a_{k}\right|+c_{k+1} \sum_{k=m+1}^{\infty}\left|a_{k}\right| \leq 1 \text {. }
$$

Finally, we can see that the left-hand side of this inequality is bounded above by the following sum:

$$
\sum_{k=2}^{\infty} c_{k}\left|a_{k}\right|
$$

which is equivalent to

$$
\sum_{k=2}^{m}\left(c_{k}-1\right)\left|a_{k}\right|+c_{k+1} \sum_{k=m+1}^{\infty}\left(c_{k}-c_{m+1}-1\right)\left|a_{k}\right| \geq 0,
$$

which is true for inequality (51).

Theorem 7. If $f$ is in the class $\mathcal{S}_{\mu}^{m, t}(\alpha, \beta, A, B)$ and has form (1), then

$$
\begin{aligned}
& \mathfrak{R}\left\{\frac{f^{\prime}(z)}{f_{m}^{\prime}(z)}\right\} \geq 1-\frac{m+1}{c_{m+1}}, \\
& \mathfrak{R}\left\{\frac{f_{m}^{\prime}(z)}{f^{\prime}(z)}\right\} \geq \frac{c_{m+1}}{(1+m)+c_{m+1}},
\end{aligned}
$$

where

$$
\begin{aligned}
c_{k}= & \frac{\Lambda_{k} \sigma_{k}}{|B-A|}, \\
& \quad c_{k} \geq\left\{1 \text { for } k=2,3, \ldots, m c_{k+1} \text { for } k=m+1, \ldots\right\} .
\end{aligned}
$$

Proof. The proof is quite the same as Theorem 6, so it is omitted.

\section{Conclusion}

The present article is the motivation of some applications of Mittag-Leffer functions in various fields of applied sciences. A subclass of analytic functions is introduced using the concepts of Pascu-type and Mittag-Leffler functions in the region of Janowski domain. Some investigations are discussed with aid of some useful properties such as sufficiency criteria, distortion and growth bounds, convex combination, radius of starlikeness, and some partial sum results. This idea can be extended to various other classes like meromorphic functions, biunivalent functions, and harmonic functions.

\section{Data Availability}

No data were used to support this study.

\section{Conflicts of Interest}

The authors declare that they have no conflicts of interest.

\section{Authors' Contributions}

All authors contributed equally to this manuscript and approved the final version.

\section{References}

[1] W. Janowski, "Some extremal problems for certain families of analytics functions," Annales Polonici Mathematici, vol. 28, pp. 297-326, 1973.

[2] M. Sabil Ur Rehman, Q. Z. Ahmad, Q. Zahoor Ahmad, H. M. Srivastava, N. Darus, and B. Khan, "Applications of higher-order q-derivatives to the subclass of q-starlike functions associated with the Janowski functions," Aims Mathematics, vol. 6, no. 2, pp. 1110-1125, 2021.

[3] S. Mehmood, Q. Z. Ahmad, H. M. Srivastava, N. Khan, B. Khan, and M. Tahir, "A certian subclass of meromorphically q-starlike functions assciated with the Janowski functions," Journal of Inequalities and Applications, vol. 2019, pp. 1-11, 2019.

[4] H. M. Srivastava, B. Khan, N. Khan, and Q. Z. Ahmad, "Coefficient inequalities for q-starlike functions associated with the Janowski functions," Hokkaido Mathematical Journal, vol. 48, pp. 407-216, 2019.

[5] H. M. Srivastava, M. Tahir, B. Khan, Q. Z. Ahmad, and N. Khan, "Some general classes of q-starlike functions associated with the Janwoski functions," Symmetry, vol. 11, pp. 1-14, 2019.

[6] H. M. Srivasatava, M. Tahir, B. Khan, Q. Z. Ahmad, and N. Khan, "Some general families of q-starlike functions associated withthe Janwoski functions," Symmetry, vol. 33, pp. 2613-2626, 2019.

[7] A. Erdelyi, W. Magnus, F. Oberhettinger, and F. G. Tricomi, Higher Transcendental Functions, Vol. 3, McGraw-Hill, New York, NY, USA, 1995.

[8] G. M. Mittag-Leffler, "Une generalisation de I'integrale de Laplace-Abel," Comptes Rendus de I'Academie des Sciences Série II, vol. 137, pp. 537-539, 1903.

[9] R. Hilfer, "Fractional diffusion based on Riemann-Liouville fractional derivatives," Journal of Physical Chemistry B, vol. 104, no. 3, pp. 914-924, 2000.

[10] G. W. S. Blair, "Psychorheology: links between the past and the present," Journal of Texture Studies, vol. 5, no. 1, pp. 3-12, 1974.

[11] H. J. Haubold, A. M. Mathai, and R. K. Sexena, "Mittag-Leffler functions and their applications," Journal of Applied Mathematics, vol. 2011, Article ID 298628, 51 pages, 2011.

[12] S. Elhaddad, H. Aldweby, and M. Darus, "On certain subclasses of analytic functions involving differential operator," Jnanabha, vol. 1, pp. 55-64, 2018.

[13] K. Vijaya, G. Murugusundaramoorthy, and M. Kasthuri, "Pascu-type harmonic functions with positive coefficients involving Salagean operator," International Journal of Analysis, vol. 2014, Article ID 793709, 10 pages, 2014.

[14] G. Murugusundaramoorthy, C. Selvaraj, and O. S. Babu, "Coefficient estimates for Pascu-type subclasses of bi- 
univalent functions based on subordination," International Journal of Nonlinear Science, vol. 19, pp. 47-52, 2015.

[15] K. Vijaya, "Certain subclass of Pascu-type bi-starlike functions in parabolic domain," Turkic World Mathematical Society Journal of Applied and Engineering Mathematics, vol. 11, pp. 580-586, 2021.

[16] H. M. Srivastava, "The Zeta and related functions: recent developments," Journal of Advanced Engineering and Computation, vol. 3, no. 1, pp. 329-354, 2019.

[17] H. M. Srivastava, S. M. El-Deeb, and S. M. El-Deeb, "The Faber polynomial expansion method and the TaylorMaclaurin coefficient estimates of Bi-Close-to-Convex functions connected with the q-convolution," AIMS Mathematics, vol. 5, no. 6, pp. 7087-7106, 2020

[18] M. S. Rehman, Q. Z. Ahmad, H. M. Srivastava, B. Khan, and N. Khan, "Partial sums of generalized $q$-Mittag-Leffer functions," Aims Mathematics, vol. 5, pp. 408-420, 2019.

[19] H. M. Srivastava and D. Bansal, "Close-to-convexity of a certain family of q-mittag-Leffler functions," Journal of Nonlinear and Variational Analysis, vol. 1, pp. 61-69, 2017.

[20] H. J. Haubold, A. M. Mathai, and R. K. Saxena, "Mittag-Leffler functions and their applications," Journal of Applied Mathematics, vol. 2011, Article ID 298628, 51 pages, 2011.

[21] M. Z. Ahmad, R. W. Ibrahim, and H. F. Al-Janaby, "On some interesting properties for a new subclass of multivalent functions," Asian-European Journal of Mathematics, vol. 9, Article ID 1650027, , 2016. 